\title{
CAPOEIRA PARA DEFICIENTES VISUAIS: COMPARAÇÃO DO EQUILÍBRIO ENTRE PRATICANTES E NÃO PRATICANTES DE CAPOEIRA
}

\author{
GRAD. JANAÍNA BARBOSA MATOS \\ Bacharel em Fisioterapia pelo Centro de Educação Física e Desportos da Universidade do Estado de \\ Santa Catarina - SC (CEFID/UDESC) (Florianópolis - Santa Catarina - Brasil) \\ Email: janafits@hotmail.com \\ MS. FÁBIO SPRADA DE MENEZES \\ Professor titular do Centro de Educação Física e Desportos (CEFID) da Universidade do Estado de \\ Santa Catarina - SC (CEFID/UDESC) (Florianópolis - Santa Catarina - Brasil) \\ Chefe do Setor de Neuroreabilitação do Centro Catarinense de Reabilitação (SES/SC) (Florianópolis \\ - Santa Catarina - Brasil) \\ Email: fabio_171I@yahoo.com.br
}

\section{RESUMO}

Objetivo: Comparar o equilíbrio de deficientes visuais praticantes e não praticantes de capoeira. Método: 10 deficientes visuais (20 a 47 anos), escolhidos de forma não probabilística intencional, capoeiristas $n=5$ e não capoeiristas $n=5$, submetidos à Escala de Equilíbrio de Berg - EEB e Escala Internacional de Eficácia de Quedas - FES-I. Resultados: A média dos valores da EEB foi de 55,4 \pm 1,34 para os capoeiristas; $54 \pm$ 1,58 para os não capoeiristas, quanto na FES-I foi de $27 \pm$ 7,64 para os capoeiristas; $35 \pm 8$, I 2 para os não capoeiristas. Conclusão: Não houve diferença estatística significativa na comparação do equilíbrio entre os praticantes e não praticantes de capoeira, embora os capoeiristas tenham obtido melhor desempenho nos dois testes aplicados.

PALAVRAS-CHAVE: Capoeira; deficientes visuais; equilíbrio; atividade física. 
A deficiência visual é um obstáculo que interfere profundamente na vida do ser humano, cujo organismo está estruturado para realizar um contínuo feedback através da visão (FIGUEIRA, 1996). No entanto, o déficit visual não impede que o indivíduo pratique atividades complexas como a Capoeira, apesar das diversas dificuldades resultantes da perda da visão, das quais ressaltamos o prejuízo no equilíbrio corporal. Ao invés disso, acredita-se que ela possa auxiliar esse indivíduo a minimizar tal déficit, uma vez que autores como Oliveira e Souza (200 I ) afirmam que na prática da Capoeira o equilíbrio é uma das qualidades mais trabalhadas.

Segundo o Instituto Brasileiro de Geografia e Estatística (IBGE), em 2000 existiam I 48 mil pessoas cegas e 2,4 milhões com grande dificuldade de enxergar. Do total de cegos, 77.900 eram mulheres e 70.100, homens.

A expressão 'deficiência visual' refere-se ao espectro que vai da cegueira até a visão subnormal (GIL, 2000), sendo essa última caracterizada pela diminuição da resposta visual, em virtude de causas congênitas ou adquirido-congênitas (MOSQUEIRA, 2000). O déficit visual traduz-se numa redução da quantidade de informação que o indivíduo recebe do meio ambiente, restringindo a grande quantidade de dados que este oferece. Consequentemente, o deficiente visual pode ter, dependendo da gravidade do déficit, um conhecimento restrito do que o rodeia (GÂNDARA, 1994).

De acordo com Skaggs e Hopper ( 1999 apud OLIVEIRA; BARRETO, 2005, p. 122), o equilíbrio em indivíduos com deficiência visual é significantemente menor que em indivíduos com visão normal. Além destes, outros autores como Andreotti e Teixeira ( 1995), Navarro et al. (2004) e Oliveira e Barreto (2005) também versam sobre o prejuízo do equilíbrio nessa população.

Ao longo dos últimos anos, o jogo da capoeira vem inserindo-se vertiginosamente nos mais diferentes espaços institucionais das médias e grandes cidades do Brasil e em vários países (FALCÃO, 2006). Hoje reconhecida como Patrimônio Cultural Brasileiro (STERF, 2008), trata-se de um misto de luta-jogo-dança praticada ao som de instrumentos musicais (berimbau, pandeiro e atabaque), palmas e cânticos. A capoeira é um excepcional sistema de auto-defesa e de condicionamento físico (FALCÃO; VIEIRA, 1997).

Os déficits de equilíbrio, de mobilidade, de coordenação motora, lateralidade e direcionalidade, esquema corporal e cinestésico do indivíduo com deficiência visual (ANDREOTTI; TEIXEIRA, 1995) são, para muitos, grandes obstáculos para a prática da Capoeira devido à exigência de respostas rápidas a golpes e movimentos inesperados. Entretanto, a capoeira possui aspectos que poderiam auxiliar o praticante 
a desenvolver mecanismos para driblar tais déficits, por utilizar movimentos que recrutam habilidades e a capacidade do corpo de se recuperar quando exposto a perturbações, aspectos estes versados por Cook e Woollacott (2003), como relacionados ao equilíbrio.

De acordo com Portugal apud Ribeiro (1992, p. 24), a capoeira proporciona melhora do tônus muscular, permite maior agilidade, flexibilidade e ampliação de movimentos. Auxilia o ajuste postural, bem como o esquema corporal, a coordenação dinâmica, a força e a ritmicidade. E, segundo Esteves (2006), sugere movimentos que influenciam diretamente na melhora do equilíbrio dinâmico, recuperando indiretamente o equilíbrio estático; deixa em estado de alerta os sistemas de proteção, ajudando na prevenção de acidentes como quedas.

A Capoeira, assim como a Educação Física em geral, adaptada a pessoas com necessidades especiais, não se diferencia da Capoeira em seus conteúdos, mas compreende técnicas, métodos e formas de organização que possam ser aplicados ao indivíduo com necessidades especiais (NEVES; FRASSON, 2003).

Este estudo buscou o desenvolvimento de ideias e descobertas para dar subsídio ao aproveitamento da Capoeira efetivamente como terapia, a fim de aprimorar o equilíbrio em indivíduos que apresentam deficiência visual, sendo que foram considerados deficientes visuais, tanto aqueles que possuem cegueira total quanto os indivíduos com baixa visão.

Apesar de muitos autores discursarem sobre os benefícios da Capoeira em diversos aspectos relacionados à motricidade humana, em especial o equilíbrio, não foram encontrados trabalhos que comparassem o equilíbrio de deficientes visuais praticantes de capoeira com o de indivíduos não praticantes de capoeira. Sendo assim, este seria o primeiro estudo com esse objetivo.

\section{MATERIAIS E MÉTODOS}

Para este estudo descritivo exploratório foram avaliados 10 indivíduos com deficiência visual, escolhidos de forma não probabilística intencional, com idades entre 20 e 47 anos, sendo que 5 deles eram praticantes de capoeira, com tempo médio de prática de 24,6 meses, em aulas ministradas duas vezes por semana I hora/aula. Desta amostra, 4 capoeiristas tiveram o primeiro contato com a prática propriamente dita no projeto em questão, intitulado "Berimbau me Guia", na ACIC (Associação Catarinense de Integração dos Cegos). Apenas um deles havia praticado capoeira quando ainda tinha a visão preservada, por um período de 6 anos.

Participaram da pesquisa 3 mulheres e 2 homens capoeiristas; e ainda 5 outros indivíduos, não praticantes de atividade esportiva, 3 homens e 2 mulheres, 
divididos então em grupo de deficientes visuais praticantes de capoeira e grupo de deficientes visuais não praticantes de capoeira.

Segundo o prontuário de identificação e procedimentos dos alunos, dos praticantes de capoeira, 2 deles apresentavam baixa visão e 3 cegueira total. Dentre as causas de baixa visão havia perda visual por sarampo, microftalmia congênita (atrofia óptica) e amaurose congênita. Dos que não praticavam atividade esportiva, 4 tinham baixa visão e apenas I cegueira total, sendo que as causas variaram entre aquisição de cegueira por toxoplasmose, microftalmia, traumatismo por golpe, e congênita por hidrocefalia.

Todos os sujeitos eram residentes na Grande Florianópolis e alunos da Associação Catarinense de Integração dos Cegos. Foram excluídas pessoas com comprometimento mental, vestibular ou proprioceptivo, e mulheres grávidas. Uma vez que o comprometimento mental pode influenciar no equilíbrio devido à percepção e habilidades viso-motoras e motoras globais estarem intrinsecamente relacionadas com substratos neurais, assim como os processos cognitivos (ROSENBAUM; CARLSON; GILMORE, 200 I; SCHWEIZER; KOCK, 2000 apud MANSUR; MARCON, 2006). Concomitantemente, Woollacott (2003) relata que, no processo de orientação vertical, empregamos múltiplas referências sensoriais, incluindo a gravidade (aparelho vestibular), a superfície de apoio (sistema somatossensitivo) e a relação do nosso corpo com o ambiente (sistema visual). Sendo assim, o comprometimento vestibular ou proprioceptivo poderia interferir nos equilíbrio dos indivíduos e, por consequência, também no resultado da pesquisa. $\bigcirc$ mesmo aconteceria em relação às mulheres grávidas, uma vez que, segundo WOLF (1993), durante a gestação ocorre mudanças físicas e o ganho de peso que determinam modificações relativas à postura, equilíbrio e locomoção.

Para avaliar o equilíbrio, foi utilizada a Escala de Equilíbrio de Berg, que atende a proposta de descrever quantitativamente a habilidade de equilíbrio funcional. Esta escala é constituída por 14 tarefas que envolvem o equilíbrio estático e dinâmico comuns à vida diária. Cada tarefa apresenta uma pontuação que varia de 0 a 4 pontos, em que 0 corresponde a equilíbrio deficiente e 4 equilíbrio excelente. Portanto, 56 pontos é a pontuação máxima. A probabilidade de queda aumenta com a diminuição da pontuação da Escala de Equilíbrio de Berg, numa relação não linear (SHUMWAY et al., 1997).

Além da EEB os sujeitos foram submetidos à avaliação sobre o medo de cair através da Escala Internacional de Eficácia de Quedas (FES-I). Nesta, o entrevistado é questionado sobre o medo de cair durante a realização de 16 atividades da vida diária, tendo como possibilidades de resposta: nenhum medo de cair (escore I), um pouco de medo de cair (escore 2), medo moderado de cair (escore 3) e muito medo de cair (escore 4). O escore total pode variar de 16 a 64, no qual o valor 16 
corresponde à ausência de preocupação e 64 preocupação extrema em relação às quedas durante a realização das atividades específicas do questionário (CAMARGOS, 2007). Para este estudo, a escala foi dividida em três partes, de acordo com o tipo de atividade: atividade de vida diária (AVD), atividade física e atividade social.

Os indivíduos foram avaliados uma única vez pela mesma examinadora, familiarizados com os testes a serem aplicados, estes escolhidos por serem funcionais, validados, aceitos internacionalmente, de fácil aplicação e de baixo custo (MYAMOTO et al., 2004). Foi usada também checagem de prontuários para confirmar se os indivíduos se encaixavam no perfil da pesquisa.

Esta pesquisa teve a aprovação do Comitê de Ética em Pesquisa em Seres Humanos da Universidade do Estado de Santa Catarina (UDESC), sob o protocolo $n^{\circ} 222 / 2008$.

\section{PROCEDIMENTOS DE COLETA}

A seleção da amostra se deu por meio de abordagem direta na própria Associação Catarinense para Integração do Cego (ACIC). Todos os indivíduos assinaram previamente um termo de consentimento. A deficiência visual foi confirmada por diagnóstico médico constado no prontuário.

A aplicação das escalas foi realizada na ACIC, em sala reservada, nos dias 28 e 29 de maio de 2009. No primeiro dia foram coletados dados dos indivíduos praticantes de capoeira, e no segundo dia os dados dos indivíduos que não praticam capoeira ou qualquer outra atividade esportiva.

\section{PROCEDIMENTOS ESTATÍSTICOS}

Foi realizada análise descritiva dos dados, através de gráficos e tabelas com média e desvio padrão dos valores da EEB e FES-I, frequência simples, percentual e dados demográficos dos indivíduos.

As comparações das avaliações aplicadas entre os grupos foram realizadas por intermédio do teste não paramétrico de Mann-Whitney, sendo considerado como o valor de significância estatística $p<0,05$.

Os dados apresentados foram analisados com o uso do software GraphPad Prism 5.0I de 2007.

\section{RESULTADOS}

Os deficientes visuais apresentaram as seguintes características: a média de idade dos capoeiristas foi de 28 anos e a média de idade dos não praticantes de 
capoeira foi de 31 , 8 anos. Os praticantes de capoeira tinham, em média, 24,6 meses de prática corridos, não se excluindo o período de férias.

A média dos escores da EEB dos praticantes de capoeira foi 55,4 \pm 1.34 pontos, e dos indivíduos não praticantes foi de $54 \pm 1.58$, e a média dos escores da FES-I foi de 27 pontos para o grupo dos capoeiristas, e 32 pontos para o grupo de não capoeiristas (Tabela I).

Segundo os dados obtidos, não houve uma diferença estatisticamente significativa entre os dois grupos para os escores da $\operatorname{EEB}(p=0,|46|)$.

Tabela I. Dados demográficos e valores da EEB E FES-I para grupo de estudo de deficientes visuais capoeiristas e não capoeiristas

\begin{tabular}{|c|c|c|c|c|c|c|c|c|c|}
\hline Grupo & Sexo & Idade & $\begin{array}{l}\text { Tempo } \\
\text { de prática } \\
\text { (meses) }\end{array}$ & $\begin{array}{l}\text { Valores } \\
\text { EEB }\end{array}$ & $\begin{array}{l}\text { Média } \\
\text { EEB }\end{array}$ & $\begin{array}{l}\text { Des- } \\
\text { vio } \\
\text { Padrão } \\
\text { EEB } \\
\end{array}$ & $\begin{array}{l}\text { Valores } \\
\text { FES-I }\end{array}$ & $\begin{array}{l}\text { Média } \\
\text { FES-I }\end{array}$ & $\begin{array}{l}\text { Desvio } \\
\text { Padrão } \\
\text { FES-I }\end{array}$ \\
\hline \multirow{5}{*}{$\begin{array}{l}\text { Capo- } \\
\text { eiristas }\end{array}$} & $\mid F$ & 22 & 27 & 56 & \multirow{5}{*}{55,4} & \multirow{5}{*}{1,34} & 33 & \multirow{5}{*}{27} & \multirow{5}{*}{7,648} \\
\hline & $2 \mathrm{~F}$ & 45 & 27 & 56 & & & 19 & & \\
\hline & $3 M$ & 25 & 27 & 56 & & & 22 & & \\
\hline & $5 \mathrm{~F}$ & 22 & 27 & 53 & & & 24 & & \\
\hline & $6 M$ & 26 & 15 & 56 & & & 37 & & \\
\hline \multirow{5}{*}{$\begin{array}{l}\text { Não } \\
\text { capo- } \\
\text { eiristas }\end{array}$} & $7 M$ & 47 & - & 55 & \multirow{5}{*}{54} & \multirow{5}{*}{1,58} & 37 & \multirow{5}{*}{32} & \multirow{5}{*}{8,124} \\
\hline & $8 M$ & 42 & - & 54 & & & 43 & & \\
\hline & $10 \mathrm{~F}$ & 23 & - & 52 & & & 29 & & \\
\hline & $\| M$ & 20 & - & 53 & & & 22 & & \\
\hline & $12 \mathrm{~F}$ & 27 & - & 56 & & & 29 & & \\
\hline
\end{tabular}

Na verificação dos resultados da FES-I, os capoeiristas demonstraram maior preocupação ou medo de cair em realizar AVD's, como preparar as refeições (muito preocupados), e vestir-se ou despir-se (um pouco preocupados). Os não capoeiristas também se preocupam com as mesmas tarefas (muito preocupados em ambas) e, além dessas, incluíram tomar banho (muito preocupados) e limpar a casa (um pouco preocupados).

Nas atividades físicas, os capoeiristas demonstraram preocupação ou medo de cair ao andar em superfícies irregulares (muito preocupados), subir e descer escadas (um pouco preocupados). Os não capoeiristas também compartilham das mesmas preocupações, acrescidas da atividade de alcançar objetos acima da cabeça (um pouco preocupados). 
Quadro I. Representação da FES-I sobre as principais preocupações dos indivíduos em realizar as atividades propostas pelo instrumento. +++ indica indivíduo muito preocupado, ++ moderadamente preocupado, + um pouco preocupado

\begin{tabular}{|c|c|c|}
\hline \multicolumn{3}{|c|}{ Representação dos Valores da FES-I } \\
\hline Atividade & Capoeiristas & Não capoeiristas \\
\hline AVD's & $\begin{array}{l}+++ \text { Preparar refeições } \\
+ \text { Vestir-se ou despir-se }\end{array}$ & $\begin{array}{l}+++ \text { Vestir-se ou despir-se } \\
+++ \text { Preparar as refeições } \\
+++ \text { Tomar banho } \\
+ \text { Limpar a casa }\end{array}$ \\
\hline Atividades físicas & $\begin{array}{l}+++ \text { Andar em superfícies ir- } \\
\text { regulares } \\
+ \text { Subir e descer escadas }\end{array}$ & $\begin{array}{l}+++ \text { Andar em superfícies irregulares } \\
+ \text { subir e descer escadas } \\
+ \text { Alcançar objetos acima da cabeça }\end{array}$ \\
\hline Atividades sociais & $\begin{array}{l}\text { + + Andar em um local onde haja } \\
\text { multidão } \\
++ \text { Sair para eventos sociais } \\
+ \text { Visitar um amigo ou parente }\end{array}$ & $\begin{array}{l}+++ \text { Andar em um local onde haja } \\
\text { multidão } \\
+++ \text { Sair para eventos sociais } \\
++ \text { Visitar um amigo ou parente }\end{array}$ \\
\hline
\end{tabular}

Nas atividades sociais, ambos preocupam-se com as mesmas atividades: andar em um local onde haja multidão, sair para eventos sociais, visitar um amigo ou parente, tendo como diferença apenas o grau de preocupação, que para os capoeiristas foi moderado nas duas primeiras atividades e pouca para a última, e, para os não capoeiristas foi de muito preocupados para duas primeiras atividades e moderado para a última, resultados esses representados no Quadro I.

Analisando os escores de cada indivíduo, observou-se que a prevalência do medo de quedas variou de 29,68\% a 57,8\% entre os deficientes visuais capoeiristas, e para os deficientes visuais não praticantes de capoeira a prevalência do medo de cair variou entre $34,37 \%$ a 67, 18\%, não havendo diferença significativa entre os dois grupos $(p=0,5284)$.

\section{Escores da FES- I}

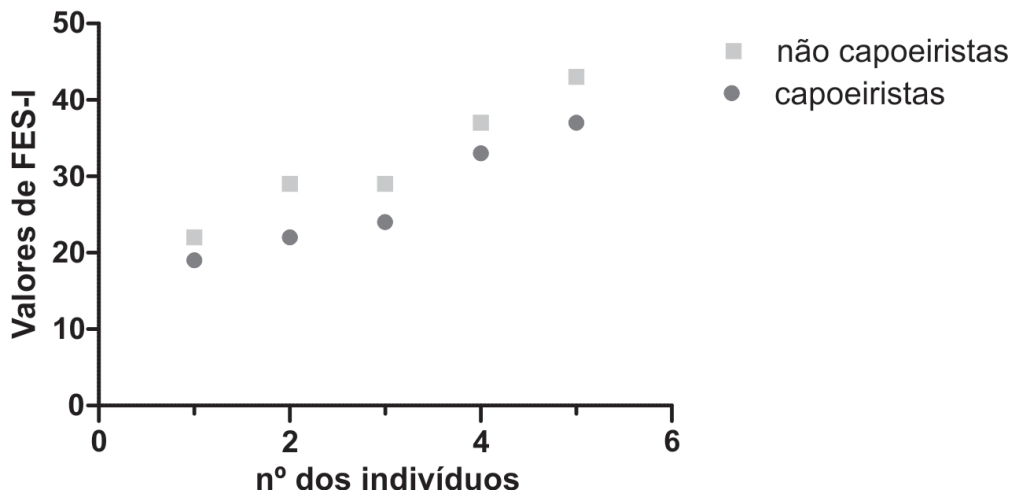

Gráfico I. Valores da FES-I evidenciam a prevalência do medo de cair dos deficientes visuais praticantes de capoeira e não praticantes. 


\section{DISCUSSÃO}

A dificuldade no equilíbrio apresentada pelos deficientes visuais, tanto praticantes de capoeira quanto não praticantes do presente estudo, corrobora com a constatação de outros autores sobre os déficit de equilíbrio e risco de queda nessa população.

Segundo Oliveira e Barreto (2005), vários estudos correlacionam a deficiência visual com alterações na função de equilíbrio. Isso ocorre porque a manutenção do controle corporal deve-se à integração de várias estruturas que compõem um sistema de controle, chamado sistema de equilíbrio. O comprometimento desse sistema pode reduzir a estabilidade, resultando em aumento da oscilação corporal e/ou alteração da estratégia de movimento (KARLSSON; FRYKBERG, 2000). Ray et al. (2008) afirma que há acréscimo no risco de quedas em indivíduos com deficiência visual. Por conseguinte, Neves e Frasson (2003) afirmam ser factível a compreensão de que os déficits motores não sejam inerentes à condição de deficiente visual, e sim, muito provavelmente, em função da limitação de experiências motoras em diversos níveis.

Alguns estudos relatam sobre o benefício de atividades físicas no equilíbrio de deficientes visuais. Silva et al. (2008) apontou que a dança proporcionou um ganho significativo, tanto no equilíbrio estático quanto no dinâmico, dos deficientes visuais do grupo experimental de I I indivíduos em uma amostra de 30 voluntários. Souza et al. (2006) estudou sobre o efeito da ginástica artística para essa mesma população, e concluiu que no final de um programa de dezoito aulas, foi possível observar melhora na capacidade física e de equilíbrio de crianças deficientes visuais.

No presente estudo, os resultados da EBB parecem indicar que os indivíduos não capoeiristas têm uma menor habilidade de equilíbrio em relação aos capoeiristas, haja visto que seus escores se distanciam mais da pontuação máxima, apesar de não haver diferença estatística significativa entre os grupos. Na FES-I ocorre o mesmo, quando mostra que os indivíduos não praticantes de capoeira apresentam-se mais temerosos em relação às quedas (Gráfico I).

Através da FES-I foi possível observar que os dois grupos se preocupam com tarefas semelhantes entre si, porém o grupo não praticante de capoeira apresenta maior preocupação em realizar AVD's e atividades físicas do que o grupo capoeirista. No entanto, em relação às atividades sociais, ambos têm um escore de preocupação muito parecido (Quadro I).

A maioria dos indivíduos (80\%) tem o mesmo tempo de prática de capoeira (27 meses), e o único indivíduo com menor tempo de prática ( 15 meses) apresenta pontuação máxima na EEB, o que indica equilíbrio excelente, assim como a maior parte dos indivíduos desse grupo. Entretanto, na FES-I este grupo obteve a maior 
pontuação, demonstrando um maior medo de cair durante as atividades, o que sugere que o tempo de prática da capoeira poderia influenciar significativamente sobre o medo de cair. No entanto, não foi possível fazer a mesma relação entre o tempo de prática e o equilíbrio dos indivíduos devido ao efeito "teto" da EEB, dito a seguir.

Possíveis hipóteses que puderam interferir nos resultado foram levantadas. Sobre limitações das escalas utilizadas: a EEB tem pontuação máxima de 56 pontos, e muitos deficientes visuais obtiveram escores que se aproximaram muito dessa pontuação, estando acima do ponto de corte estabelecido por Berg et al. (1989) e Chiu et al. (2003) de 45 e 38, respectivamente, para determinação de indivíduos com mais chances de queda. No entanto, tais parâmetros foram determinados em estudos aplicados em idosos saudáveis. Outros autores, como Belgen et al. (2006), assinalam que o ponto de corte de 52 na EEB é o mais adequado como indicador de quedas em hemiparéticos, após análise de 50 vítimas de AVE na fase crônica, ou seja, dependendo da população em estudo, o ponto de corte pode variar para conferir maior especificidade à aplicação da escala. Uma vez que não há estudos que adequem o ponto de corte para a EEB na averiguação do equilíbrio em deficientes visuais, pôde-se observar que esta escala parece não ser sensível o bastante para detectar alteração no equilíbrio de deficientes visuais.

Além disso, não havia muitos deficientes visuais praticantes de capoeira que se encaixassem no perfil do estudo, o que resultou em uma amostra pequena e bastante heterogênea no que diz respeito aos diferentes graus de deficiência visual em ambas as amostras, que variaram de cegueira total à visão subnormal ou baixa visão.

Devido à escassez de testes para quantificar o risco de quedas, principalmente para a população deste estudo, alguns autores sugerem o aperfeiçoamento dos existentes e a criação de novos testes (RESENDE et al., 2008). Além disso, novos estudos, com amostras mais representativas, poderiam auxiliar na busca de resultados irrefutáveis na comparação do equilíbrio entre deficientes visuais praticantes e não praticantes de Capoeira.

\section{CONCLUSÃO}

Após a análise dos resultados deste estudo, observou-se que a Capoeira não melhorou o equilíbrio dos deficientes visuais, embora os capoeiristas tenham obtido melhor desempenho nos dois testes aplicados. Sugere-se a realização de novos estudos, com amostras mais representativas e instrumentos mais sensíveis a essa população, para corroborar ou não com os achados. 


\section{Capoeira for visually impaired: comparison of the balance between practitioners and non-practitioners of Capoeira.}

ABSTRACT: Purpose: To compare the balance between visually impaired practitioners of Capoeira and non-practitioners of Capoeira. Methods: I 0 visually impaired individuals (20 to 47 years), chosen on a non-probabilistic intentional, capoeirists $n=5$ and $n=5$ non capoeirists, submitted to the Berg Balance Scale (BBE) and Falls Efficacy Scale (FES-I). Results: The BBE average points was $55.4 \pm 1.34$ for capoeirists and $54 \pm 1.58$ for non-capoeirists, and the average result obtained in the FES-I was $27 \pm 7.64$ for capoeirists and $35 \pm 8.12$ for non-capoeirists. Conclusion: There were not significant statistic differences in the balance between the two groups, although the capoeirists have had better scores in both tests.

KEYWORDS: Capoeira; visual impairement; balance; physical activity.

Capoeira para los discapacitados visuales: comparación del equilibrio de los practicantes y no practicantes de la capoeira.

RESUMEN: Finalidad: Comparar el equilibrio entre los discapacitados visuales practicantes de la Capoiera y no practicantes de la Capoeira. Métodos: 10 discapacitados visuales (20 a 47 años), elegidos de forma no probabilística intencional, capoeiristas $n=5$ y $n=5$ no capoeiristas, presentado a la Berg Escala del equilibrio - EEB y la Escala de Eficácia de Quedas - FES-I. Resultados: La media de los puntos de la EEB fue 55,4 \pm 1,34 para los capoeiristas y $54 \pm 1,58$ para los no capoeiristas, y la media de los resultados obtenidos en la FES-I se $27 \pm 7,64$ para los capoeiristas y $35 \pm 8,12$ para los no capoeiristas. La diferencia entre los grupos en la incidencia de EEB fue $p=0,146$ y FES-I fue $p=0,5284$. Conclusión: La comparación del saldo a partir de las puntuaciones de los individuos en las escalas, no se observaron diferencias significativas en el rendimiento entre los dos grupos, a pesar de que capoeiristas han obtenido mejores resultados en ambas las escalas.

PALABRAS CLAVE: Capoeira; discapacitados visuales; equilíbrio; actividad física.

\section{REFERÊNCIAS}

ANDREOTTI, R. A.; TEIXEIRA, L. R. O papel da educação física adaptada no desenvolvimento motor do indivíduo portador de deficiência visual. Revista Brasileira de Saúde Escolar, Campinas, v. 3, n. I-4, p. 76-82, 1995.

BELGEN, B. et al. The association of balance capacity and falls self-efficacy with history of falling in community-dwelling people with chronic stroke. Physical Medicine and Rehabilitation, Philadelphia, v. 87, n. 4, p. 554-56I, apr. 2006.

BERG, K. O. et al. Measuring balance in the elderly: preliminary development of an instrument. Physiotherapy, Toronto, v. 4I, n. 6, p. 304-3II, nov./dec. 1989.

BRENNECKE, A.; AMADIO, A. C.; SERRAO, J. C. Parâmetros dinâmicos de movimentos selecionados da Capoeira. Revista Portuguesa de Ciências do Desporto, Porto, v. 5, n. 2, p. I53- I 59, maio 2005. 
BUENO, J. M. Psicomotricidade teoria e prática: estimulação, educação e reeducação. São Paulo: Lovise, 1998.

CAMARGOS, F. F. O. Adaptação transcultural e avaliação das propriedades psicométricas da falls efficacy scale - international: um instrumento para avaliar medo de cair em idosos. 2007. Dissertação (Mestrado) - Escola de Educação Física, Fisioterapia e Terapia Ocupacional, Universidade Federal de Minas Gerais, Belo Horizonte, 2007.

CHIU, A. Y.; AU, Y.; LO, S. K. A comparison of four functional tests in discriminating fallers from non-fallers in older people. Disability and Rehabilitation, London, v. 25, n. I, p. 45-50, jan. 2003.

COOK, A. S.; WOOLLACOTT, M. H. Controle motor: teorias e aplicações práticas. Barueri: Manole, 2003.

ESTEVES, A. A Capoeira como atividade terapêutica: novas possibilidades de reabilitação. Jornal do Capoeira, Salvador, n. 55, jan. 2005. Disponível em: <http://74.125.93.132/ search?q=cache:7WHQx64F9all:www.capoeira.jex.com.br/noticias/detalhes.php\%3Fid jornal\%3D 13 | 70\%26id_noticia\%3D778+\%5DESTEVES + Capoeira + como + atividade + terap\%C3\%AAutica. + Jornal + do + Capoeira. +Edi\%C3\%A7\%C3\%A3o + 55 +- + de +08/

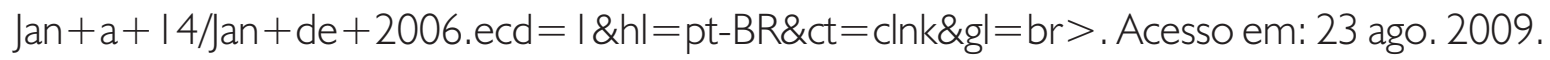

FALCÃO, J. L. C. O jogo da capoeira em jogo. Revista Brasileira de Ciências do Esporte, Campinas, v. 27, n. 2, p. 59-74, jan. 2006.

FIGUEIRA, M. M. A. Assistência fisioterápica à criança portadora de cegueira congênita. Revista Benjamin Constant, Rio de Janeiro, n. 5, p. 8-23, dez. 1996.

GÂNDARA, M. A Expressão corporal do deficiente visual. Campinas: Ed. da Unicamp, 1994.

GIL, M. Deficiência visual. Cadernos da Tv Escola. Brasília, 2000. Disponível em: <http:// portal.mec.gov.br/seed/arquivos/pdf/deficienciavisual.pdf> Acesso em: I I jun. 2009.

INSTITUTO BRASILEIRO DE GEOGRAFIA E ESTATIÍSTICA (IBGE). Censo demográfico do ano 2000. Disponível em: <http://www.ibge.gov.br>. Acesso em: 2 I ago. 20l I.

KARLSSON, A.; FRYKBERG, G. Correlations between force plate measures for assessment of balance. Clinical Biomechanics, Oxford, v. I5, 5, p. 365-369, jun. 2000.

MANSUR, S. S.; MARCON, A. J. Perfil motor de crianças e adolescentes com deficiência mental moderada. Revista Brasileira de Crescimento e Desenvolvimento Humano, São Paulo, v. 16, n. 3, p. 9-15, 2006.

MOSQUEIRA, C. Educação física para deficientes visuais. Rio de Janeiro: Sprint, 2000.

MIYAMOTO, S. T. et al. Brazilian version of the Berg balance scale. Brazilian Journal of Medical and Biological Research, Ribeirão Preto, v. 37, n. 9, p. I4 I I- | 42 I, sept. 2004.

NAVARRO, A. S. et al. Balance and motor coordination are not fully developed in 7 years old blind children. Arquivos de Neuro-Psiquiatria, São Paulo, v. 62, n. 3a, sept. 2004. Disponível 
em: <http://www.scielo.br/scielo.php?script=sci_arttext\&pid=S0004-282X20040004000 l6\&lng=en\&nrm=iso >. Acesso em: 22 jun. 2009.

NEVES, G. N.; FRASSON, A. C. Educação Física adaptada ao deficiente visual. In: SIMPÓSIO DE EDUCAÇÃO FÍSICA E DESPORTOS DO SUL DO BRASIL, I 5., Ponta Grossa, 2003. Anais... Jundiaí: Fontoura, 2003. p. 291-291.

OLIVEIRA, D. N.; BARRETO, R. R. Avaliação do equilíbrio estático em deficientes visuais adquiridos. Revista Neurociências, São Paulo, v. 13, n. 3, p. I22-127, jul./set. 2005.

OLIVEIRA, A. A. B.; SOUZA, S. A. R. Estruturação da capoeira como conteúdo da Educação Física. Revista da Educação Física, Maringá, v. I2, n. 2, p. 43-50, 2001.

RAY, C. et al. The impact of vision loss on postural stability and balance strategies in individuals with profound vision loss. Gait \& Posture, Oxford, v. 28, n. I, p. 58-6I, 2008.

RESENDE, S. V.; RASSI, C. M.; VIANA, F. P. Efeitos da hidroterapia na recuperação do equilíbrio e prevenção de quedas em idosas. Revista Brasileira de Fisioterapia, São Carlos, v. I2, n. I, p. 57-63, jan./fev. 2008.

RIBEIRO, A. L. Capoeiraterapia. 3. ed. Brasília: Secretaria dos Desportos, 1992.

ROSENBAUM, D. A.; CARLSON, R. A.; GILMORE, R. O. Acquisition of intellectual and perceptual-motor skills. Annual Review of Psychology, Palo Alto, v. 52, p. 453-470, 2001.

SCHEWEIZER, K.; KOCK, W. Perceptual processes and cognitive ability. Intelligence, v. 3 I, n. 3, p. $21 \mid-235,2000$.

SHUMWAY-COOK, A. et al. Predicting the Probability for falls in community-dwelling older adults. Physical Therapy, Alexandria, v. 77, n. 8, p. 812-819, 1997. Disponível em: <http:// physicaltherapyonline.net/cgi/reprint/77/8/8 I2.pdf>. Acesso em: 30 maio 2009.

SILVA, C. A.; RIBEIRO, G. M.; RABELO, R. J. A influência da dança no equilíbrio corporal de deficientes visuais. Movimentum: Revista Digital de Educação Física, Ipatinga, v. 3, n. I, p. I-8, fev./jul. 2008. Disponível em: <http://www.unilestemg.br/movimentum/Artigos_V3NI_em_pdf/movimentum_v3_nl_silva_cristiane_ribeiro_graziele_2_2007.pdf > Acesso em: 21 ago. 201 l.

SKAGGS, S.; HOPPER, C. Individuals with visual impairments: a review of psychomotor behavior. Adapted Physical Activity Quarterly, Champaign, v. 13, p. 16-26, 1999.

SOUZA, C. M. et al. Ginástica Artística para crianças deficientes visuais: relato de experiência. EF Deportes: revista digital, Buenos Aires, ano 10, n. 94, mar. 2006. Disponível em: < http:// webcache.googleusercontent.com/search?q=cache: I Fw3M I cO6Po):www.efdeportes.com/ efd94/visual.htm $+\& c d=\mid \& h l=p t-B R \& c t=c l n k \&$ source $=$ encrypted.google.com $>$. Acesso em: 2 I ago. 201 I.

STERF, S. Iphan registra capoeira como patrimônio cultural brasileiro. Folha.com. Disponível em: http://64.233. I69. I04/search?q=cache:aGoaTgo6vYkJ:www.cultura.gov.br/ 
site/2008/07/I 5/iphan-registra-capoeira-como-patrimonio-cultural-brasileiro/+ capoeira + patri $\mathrm{m} \% \mathrm{C3} \% \mathrm{~B} 4 \mathrm{nio}+$ cultural + minist\%C3\%A9rio $+\mathrm{da}+$ Cultura\&hl $=\mathrm{pt}-\mathrm{BR} \& \mathrm{ct}=\mathrm{clnk} \& \mathrm{~cd}=2 \& \mathrm{~g} \mid=\mathrm{br}$. Acesso em: 30 maio 2009.

THORBAHN, L. D.; NEWTON, R. A. Use of the Berg Balance Test to predict falls in elderly persons. Physical Therapy, Alexandria, v. 76, n. 6, p. 576-585, jun. 1996.

WOLF, L. A. Pregnancy. In: SKINNER, J. S. Exercise testing and exercise prescription for special cases: theoreticals basis and clinical application. Philadelphia: Lea and Febiger, I 993. Disponível em: <http://www.ncbi.nlm.nih.gov/pmc/articles/PMCI 478467/>. Acesso em: 29 jun. 20 I I.

WOOLLACOTT, M. H. Controle postural normal. In: SHUMUAY-COOK, A. S.; WOOLLACOTT, M. Controle motor: teorias e aplicações práticas. São Paulo: Manole, 2003. p. I 53-I 78. ; TANG, P. Balance control during walking in the older adult: research and its implications. Physical Therapy, Alexandria v. 77, n. 6, p. 646-660, jun. 1997.

Recebido: 30 set. 2009

Aprovado: I 2 ago. 20 I I

Endereço para correspondência Janaína Barbosa Matos R. T-64A, 136, ap. II05 Setor Bela Vista Goiânia - GO CEP: 74823360 